Supporting Information

\title{
Metagenomic sequencing revealed the potential pathogenic threats of banknotes
}

Jun Lin ${ }^{1,2,3,4, \dagger}$, Wenqian Jiang ${ }^{1,3, \dagger}$, Yang Shi ${ }^{1,3}$, Weiwen Cai $1^{1,3^{*}}$

${ }^{1}$ Institute of Applied Genomics, Fuzhou University, Fuzhou, China

${ }^{2}$ School of Basic Medical Sciences, Fujian Medical University, Fuzhou, China

${ }^{3}$ College of Biological Science and Engineering, Fuzhou University, Fuzhou, China

${ }^{4}$ Fujian Key Laboratory of Marine Enzyme Engineering, Fuzhou University, No.2

Xueyuan Road, Fuzhou, China

* Correspondence:

Weiwen Cai

caiww@,fzu.edu.cn

$\dagger$ These authors contributed equally to the work 
Scheme S1

\section{STE DNA extraction method}

1. Banknotes were rinsed with distilled water, which was collected and passed through a filter membrane. The filter membrane was ground up in a $2 \mathrm{ml}$ tube with a micro pestle in $600 \mu \mathrm{l}$ X STE buffer $(50 \mathrm{mM} \mathrm{NaCl}, 50 \mathrm{mM}$ Tris-HCL, 100 mM EDTA, $\mathrm{pH} 8.0)$ along with $60 \mu \mathrm{l}$ lysozyme $(200 \mathrm{mg} / \mathrm{ml})$. The mixture was incubated for 30 minutes at $37{ }^{\circ} \mathrm{C}$.

2. Add 1\% SDS, $6 \mu \mathrm{l}$ RNase A $(20 \mathrm{mg} / \mathrm{ml}), 12 \mu$ Proteinase $\mathrm{K}(20 \mathrm{mg} / \mathrm{ml})$ and mix it.

3. Lyse for 30 minutes at $65{ }^{\circ} \mathrm{C}$. Gently mix the tube by inverting every 5 minutes.

4. Centrifuge at $12,000 \mathrm{~g}$ for 10 minutes at $4{ }^{\circ} \mathrm{C}$. Transfer the supernatant to a fresh tube.

5. Add equal volume of Tris saturated phenol mix and centrifuge at $12,000 \mathrm{~g}$ for 10 minutes at $4{ }^{\circ} \mathrm{C}$. Transfer the supernatant to a fresh tube.

6. Then add equal volume of chloroform: isoamylalcohol (24:1) and centrifuge at $12,000 \mathrm{~g}$ for 10 minutes at $4{ }^{\circ} \mathrm{C}$.

7. Transfer the clear supernatant to a fresh tube, add two fold volume cold ethanol and keep it for 1 hour at $-20{ }^{\circ} \mathrm{C}$.

8. Centrifuge at $12,000 \mathrm{~g}$ for 10 minutes at $4{ }^{\circ} \mathrm{C}$ and then remove the supernatant.

9. Wash the pellet with $70 \%$ ethanol.

10. Keep the pellet at $37{ }^{\circ} \mathrm{C}$ for 10 minutes.

11. Dissolve the dry pellet in $100 \mu 1$ nuclease free water. Store the isolated DNA 
at $-20^{\circ} \mathrm{C}$.

\section{Sequencing}

A total amount of $1 \mu \mathrm{g}$ metagenomic DNA per sample was used as input material for preparation of DNA libraries. Sequencing libraries were generated using NEBNext ${ }^{\circledR}$ Ultra $^{\mathrm{TM}}$ DNA Library Prep Kit for an Illumina Hiseq2500 sequencer (NEB, USA) following manufacturer's recommendations and index codes were added to mark sequences for each sample. Briefly, the DNA sample was fragmented by sonication to an average size of $300 \mathrm{bp}$, then DNA fragments were end-polished, A-tailed, and ligated with the full-length adaptor for Illumina sequencing with further PCR amplification. PCR products were purified (AMPure XP system) and libraries were analyzed for size distribution by Agilent 2100 Bioanalyzer and quantified using real-time PCR. An Illumina Hiseq2500 sequencer was used for high-throughput sequencing of the four DNA samples and paired-end reads were generated. 
Scheme S2

\section{Informatic analysis of metagenomes of banknotes}

Metagenomic analysis can more truly reflect the microbial composition and interaction in the sample, and study the metabolic pathway and gene function at the molecular level. ${ }^{1-4}$.

1. Preprocessing of sequencing data

The Raw Data obtained from the Illumina HiSeq sequencing platform was preprocessed using Readfq (https://github.com/lh3/readfq) to obtain the Clean Data for subsequent analysis.

(1) Remove reads that contain low-quality bases (mass value $\leqslant 5$ ) that exceed a certain percentage (default is $40 \%$ of the length of reads);

(2) Remove reads that $\mathrm{N}$ bases achieve a certain percentage (default is $10 \%$ of the length of reads);

(3) Remove the reads whose overlap with adapter exceeds threshold (default value is $15 \mathrm{bp}$ );

(4) If the sample has host contamination, it needs to be compared with the host database to filter out reads that may originate from the host (default consistency $\geqslant 90 \%)$.

\section{Metagenome Assembly}

The Clean Data was assembled and analyzed using the SOAPdenovo software ${ }^{5}$ with parameters ${ }^{6-9}$ set as follows: $-\mathrm{d} 1,-\mathrm{M} 3,-\mathrm{R},-\mathrm{U},-\mathrm{F},-\mathrm{K} 55$; then split the assembled Scaftigs at $\mathrm{N}$ and to generate Scaftigs without $\mathrm{N}^{7,10-11}$.

3. Taxonomy prediction

1) The USEARCH software was used to reduce the redundancy of Scaftigs from each sample ${ }^{12}$. The Scaftigs were blasted against the sequences of Bacteria, Fungi, Archaea and Viruses, which were all extracted from the NT database (Version: 201410-19, https: //www.ncbi.nlm.nih.gov/) of NCBI with the parameter setting as blastp, -e 1e-5.

2) For the finally aligned results of each sequence, as each sequence may have 
multiple aligned results, we used the LCA algorithm, the software used in MEGAN 13 for system classification, to annotate the sequences for the species assignment. 3) The table containing the number of genes and the abundance information of each sample in each taxonomy hierarchy (kingdom, phylum, class, order, family, genus, species) were obtained based on the LCA annotation results and the gene abundance table. Command: draw_tree.pl--trantab -bline --scal_title 'BrayCurtis Distance' -width 200 -type 3 -bun 0.25, 4 -btitle 'Relative Abundance in Order Level' Unigenes.readsNum.even.tree table.o10.cluster.xls --group > Bar.tree.010.svg.

4. Gene prediction and abundance analysis

1) The ORF of assembled Scaftigs ( $\geq 300 \mathrm{bp}$ ) ${ }^{14}$ were fed into MetaGeneMark ${ }^{15}$ software for gene prediction.

2) For the predicted ORFs, CD-HIT ${ }^{16-17}$ software was used to remove redundant predictions and obtain the unique initial gene catalogue ${ }^{18}$, the parameters option 18-19 are -c 0.95, -G 0, -aS 0.9, -g 1, -d 0.

3) Based on the number of mapped reads and the gene length, the abundance information of each gene in each sample was calculated using the formula as follows:

$$
G_{k}=\frac{r_{k}}{L_{k}} \cdot \frac{1}{\sum_{i=1}^{n} \frac{r_{i}}{L_{i}}}
$$

where $r$ represents the number of reads mapped to the genes and $L$ represents gene's length ${ }^{19-24}$.

4) The basic information statistics, core-pan gene analysis, correlation analysis of samples and Venn figure analysis of number of genes are all based on the abundance of each gene in each sample in gene catalogue.

5. Common functional database annotations 
1) Unigenes blasted against a functional database with the parameter setting of blastp, -e 1e-5 ${ }^{9}$. 25 . Functional databases include KEGG ${ }^{26-27}$ database (Version 58, http://www.kegg.jp/kegg/), eggNOG 28 database (Version 4.0, http://eggnogdb.embl.de/\#/app/home), CAZy ${ }^{29}$ database (Version 20141125, http://www.cazy.org/). For each sequence's blast result, the best Blast Hit is used for subsequent analysis $9,25,30$.

2) Statistics of the relative abundance of different functional hierarchy. The relative abundance of each functional hierarchy equals the sum of relative abundance annotated to that functional level.

3) Based on the function annotation result and gene abundance table, the gene number table of each sample in each taxonomy hierarchy is obtained.

Readfq (V5, https://github.com/cjfields/readfq)

SOAP denovo (Version: 2.21): http://soap.genomics.org.cn/soapdenovo.html

USEARCH (Version: 7.0.1001):

http://www.drive5.com/usearch/manual/dereplication.html

SoapAligner (Version: 2.21): http://soap.genomics.org.cn/soapaligner.html

$\begin{array}{lll}\text { MetaGeneMark } & \text { 2.10): }\end{array}$

http://exon.gatech.edu/GeneMark/metagenome/Prediction

CD-HIT (Version: 4.5.8): http://www.bioinformatics.org/cd-hit/

\section{References}

1. Chen, K.; Pachter, L., Bioinformatics for whole-genome shotgun sequencing of microbial communities. PLoS Comput Biol 2005, 1 (2), 106-12.

2. Handelsman, J.; Rondon, M. R.; Brady, S. F.; Clardy, J.; Goodman, R. M., Molecular biological access to the chemistry of unknown soil microbes: a new frontier for natural products. Chem Biol 1998, 5 (10), R245-9.

3. Tringe, S. G.; Rubin, E. M., Metagenomics: DNA sequencing of environmental samples. Nat Rev Genet 2005, 6 (11), 805-14.

4. Tringe, S. G.; von Mering, C.; Kobayashi, A.; Salamov, A. A.; Chen, K.; Chang, H. W.; Podar, M.; Short, J. M.; Mathur, E. J.; Detter, J. C.; Bork, P.; Hugenholtz, P.; Rubin, E. M., Comparative metagenomics of microbial communities. Science 2005, $308(5721), 554-7$. 
5. Luo, R.; Liu, B.; Xie, Y.; Li, Z.; Huang, W.; Yuan, J.; He, G.; Chen, Y.; Pan, Q.; Liu, Y.; Tang, J.; Wu, G.; Zhang, H.; Shi, Y.; Liu, Y.; Yu, C.; Wang, B.; Lu, Y.; Han, C.; Cheung, D. W.; Yiu, S. M.; Peng, S.; Xiaoqian, Z.; Liu, G.; Liao, X.; Li, Y.; Yang, H.; Wang, J.; Lam, T. W.; Wang, J., SOAPdenovo2: an empirically improved memoryefficient short-read de novo assembler. Gigascience 2012, 1 (1), 18.

6. Scher, J. U.; Sczesnak, A.; Longman, R. S.; Segata, N.; Ubeda, C.; Bielski, C.; Rostron, T.; Cerundolo, V.; Pamer, E. G.; Abramson, S. B.; Huttenhower, C.; Littman, D. R., Expansion of intestinal Prevotella copri correlates with enhanced susceptibility to arthritis. Elife 2013, 2, e01202.

7. Qin, N.; Yang, F.; Li, A.; Prifti, E.; Chen, Y.; Shao, L.; Guo, J.; Le Chatelier, E.; Yao, J.; Wu, L.; Zhou, J.; Ni, S.; Liu, L.; Pons, N.; Batto, J. M.; Kennedy, S. P.; Leonard, P.; Yuan, C.; Ding, W.; Chen, Y.; Hu, X.; Zheng, B.; Qian, G.; Xu, W.; Ehrlich, S. D.; Zheng, S.; Li, L., Alterations of the human gut microbiome in liver cirrhosis. Nature 2014, 513 (7516), 59-64.

8. Brum, J. R.; Ignacio-Espinoza, J. C.; Roux, S.; Doulcier, G.; Acinas, S. G.; Alberti, A.; Chaffron, S.; Cruaud, C.; de Vargas, C.; Gasol, J. M.; Gorsky, G.; Gregory, A. C.; Guidi, L.; Hingamp, P.; Iudicone, D.; Not, F.; Ogata, H.; Pesant, S.; Poulos, B. T.; Schwenck, S. M.; Speich, S.; Dimier, C.; Kandels-Lewis, S.; Picheral, M.; Searson, S.; Tara Oceans, C.; Bork, P.; Bowler, C.; Sunagawa, S.; Wincker, P.; Karsenti, E.; Sullivan, M. B., Ocean plankton. Patterns and ecological drivers of ocean viral communities. Science 2015, 348 (6237), 1261498.

9. Feng, Q.; Liang, S.; Jia, H.; Stadlmayr, A.; Tang, L.; Lan, Z.; Zhang, D.; Xia, H.; Xu, X.; Jie, Z.; Su, L.; Li, X.; Li, X.; Li, J.; Xiao, L.; Huber-Schonauer, U.; Niederseer, D.; Xu, X.; Al-Aama, J. Y.; Yang, H.; Wang, J.; Kristiansen, K.; Arumugam, M.; Tilg, H.; Datz, C.; Wang, J., Gut microbiome development along the colorectal adenomacarcinoma sequence. Nat Commun 2015, 6, 6528.

10. Mende, D. R.; Waller, A. S.; Sunagawa, S.; Jarvelin, A. I.; Chan, M. M.; Arumugam, M.; Raes, J.; Bork, P., Assessment of metagenomic assembly using simulated next generation sequencing data. PloS one 2012, 7 (2), e31386.

11. Nielsen, H. B.; Almeida, M.; Juncker, A. S.; Rasmussen, S.; Li, J. H.; Sunagawa, S.; Plichta, D. R.; Gautier, L.; Pedersen, A. G.; Le Chatelier, E.; Pelletier, E.; Bonde, I.; Nielsen, T.; Manichanh, C.; Arumugam, M.; Batto, J. M.; dos Santos, M. B. Q.; Blom, N.; Borruel, N.; Burgdorf, K. S.; Boumezbeur, F.; Casellas, F.; Dore, J.; 
Dworzynski, P.; Guarner, F.; Hansen, T.; Hildebrand, F.; Kaas, R. S.; Kennedy, S.; Kristiansen, K.; Kultima, J. R.; Leonard, P.; Levenez, F.; Lund, O.; Moumen, B.; Le Paslier, D.; Pons, N.; Pedersen, O.; Prifti, E.; Qin, J. J.; Raes, J.; Sorensen, S.; Tap, J.; Tims, S.; Ussery, D. W.; Yamada, T.; Renault, P.; Sicheritz-Ponten, T.; Bork, P.; Wang, J.; Brunak, S.; Ehrlich, S. D.; Consortium, M., Identification and assembly of genomes and genetic elements in complex metagenomic samples without using reference genomes. Nature Biotechnology 2014, 32 (8), 822-828.

12. Edgar, R. C., Search and clustering orders of magnitude faster than BLAST. Bioinformatics 2010, 26 (19), 2460-2461.

13. Huson, D. H.; Mitra, S.; Ruscheweyh, H. J.; Weber, N.; Schuster, S. C., Integrative analysis of environmental sequences using MEGAN4. Genome Res 2011, 21 (9), 15521560.

14. Yok, N. G.; Rosen, G. L., Combining gene prediction methods to improve metagenomic gene annotation. BMC Bioinformatics 2011, 12, 20.

15. Zhu, W.; Lomsadze, A.; Borodovsky, M., Ab initio gene identification in metagenomic sequences. Nucleic acids research 2010, 38 (12), e132.

16. Li, W.; Godzik, A., Cd-hit: a fast program for clustering and comparing large sets of protein or nucleotide sequences. Bioinformatics 2006, 22 (13), 1658-9.

17. Fu, L.; Niu, B.; Zhu, Z.; Wu, S.; Li, W., CD-HIT: accelerated for clustering the next-generation sequencing data. Bioinformatics 2012, 28 (23), 3150-2.

18. Sunagawa, S.; Coelho, L. P.; Chaffron, S.; Kultima, J. R.; Labadie, K.; Salazar, G.; Djahanschiri, B.; Zeller, G.; Mende, D. R.; Alberti, A.; Cornejo-Castillo, F. M.; Costea, P. I.; Cruaud, C.; d'Ovidio, F.; Engelen, S.; Ferrera, I.; Gasol, J. M.; Guidi, L.; Hildebrand, F.; Kokoszka, F.; Lepoivre, C.; Lima-Mendez, G.; Poulain, J.; Poulos, B. T.; Royo-Llonch, M.; Sarmento, H.; Vieira-Silva, S.; Dimier, C.; Picheral, M.; Searson, S.; Kandels-Lewis, S.; Tara Oceans, c.; Bowler, C.; de Vargas, C.; Gorsky, G.; Grimsley, N.; Hingamp, P.; Iudicone, D.; Jaillon, O.; Not, F.; Ogata, H.; Pesant, S.; Speich, S.; Stemmann, L.; Sullivan, M. B.; Weissenbach, J.; Wincker, P.; Karsenti, E.; Raes, J.; Acinas, S. G.; Bork, P., Ocean plankton. Structure and function of the global ocean microbiome. Science 2015, 348 (6237), 1261359.

19. Zeller, G.; Tap, J.; Voigt, A. Y.; Sunagawa, S.; Kultima, J. R.; Costea, P. I.; Amiot, A.; Bohm, J.; Brunetti, F.; Habermann, N.; Hercog, R.; Koch, M.; Luciani, A.; Mende, D. R.; Schneider, M. A.; Schrotz-King, P.; Tournigand, C.; Tran Van Nhieu, J.; 
Yamada, T.; Zimmermann, J.; Benes, V.; Kloor, M.; Ulrich, C. M.; von Knebel Doeberitz, M.; Sobhani, I.; Bork, P., Potential of fecal microbiota for early-stage detection of colorectal cancer. Mol Syst Biol 2014, 10, 766.

20. Qin, J.; Li, R.; Raes, J.; Arumugam, M.; Burgdorf, K. S.; Manichanh, C.; Nielsen, T.; Pons, N.; Levenez, F.; Yamada, T.; Mende, D. R.; Li, J.; Xu, J.; Li, S.; Li, D.; Cao, J.; Wang, B.; Liang, H.; Zheng, H.; Xie, Y.; Tap, J.; Lepage, P.; Bertalan, M.; Batto, J. M.; Hansen, T.; Le Paslier, D.; Linneberg, A.; Nielsen, H. B.; Pelletier, E.; Renault, P.; Sicheritz-Ponten, T.; Turner, K.; Zhu, H.; Yu, C.; Li, S.; Jian, M.; Zhou, Y.; Li, Y.; Zhang, X.; Li, S.; Qin, N.; Yang, H.; Wang, J.; Brunak, S.; Dore, J.; Guarner, F.; Kristiansen, K.; Pedersen, O.; Parkhill, J.; Weissenbach, J.; Meta, H. I. T. C.; Bork, P.; Ehrlich, S. D.; Wang, J., A human gut microbial gene catalogue established by metagenomic sequencing. Nature 2010, 464 (7285), 59-65.

21. Karlsson, F. H.; Fak, F.; Nookaew, I.; Tremaroli, V.; Fagerberg, B.; Petranovic, D.; Backhed, F.; Nielsen, J., Symptomatic atherosclerosis is associated with an altered gut metagenome. Nature Communications 2012, 3.

22. Cotillard, A.; Kennedy, S. P.; Kong, L. C.; Prifti, E.; Pons, N.; Le Chatelier, E.; Almeida, M.; Quinquis, B.; Levenez, F.; Galleron, N.; Gougis, S.; Rizkalla, S.; Batto, J. M.; Renault, P.; consortium, A. N. R. M.; Dore, J.; Zucker, J. D.; Clement, K.; Ehrlich, S. D., Dietary intervention impact on gut microbial gene richness. Nature 2013, 500 (7464), 585-8.

23. Le Chatelier, E.; Nielsen, T.; Qin, J.; Prifti, E.; Hildebrand, F.; Falony, G.; Almeida, M.; Arumugam, M.; Batto, J. M.; Kennedy, S.; Leonard, P.; Li, J.; Burgdorf, K.; Grarup, N.; Jorgensen, T.; Brandslund, I.; Nielsen, H. B.; Juncker, A. S.; Bertalan, M.; Levenez, F.; Pons, N.; Rasmussen, S.; Sunagawa, S.; Tap, J.; Tims, S.; Zoetendal, E. G.; Brunak, S.; Clement, K.; Dore, J.; Kleerebezem, M.; Kristiansen, K.; Renault, P.; Sicheritz-Ponten, T.; de Vos, W. M.; Zucker, J. D.; Raes, J.; Hansen, T.; Meta, H. I. T. c.; Bork, P.; Wang, J.; Ehrlich, S. D.; Pedersen, O., Richness of human gut microbiome correlates with metabolic markers. Nature 2013, 500 (7464), 541-6.

24. Villar, E.; Farrant, G. K.; Follows, M.; Garczarek, L.; Speich, S.; Audic, S.; Bittner, L.; Blanke, B.; Brum, J. R.; Brunet, C.; Casotti, R.; Chase, A.; Dolan, J. R.; d'Ortenzio, F.; Gattuso, J. P.; Grima, N.; Guidi, L.; Hill, C. N.; Jahn, O.; Jamet, J. L.; Le Goff, H.; Lepoivre, C.; Malviya, S.; Pelletier, E.; Romagnan, J. B.; Roux, S.; Santini, S.; Scalco, E.; Schwenck, S. M.; Tanaka, A.; Testor, P.; Vannier, T.; Vincent, F.; Zingone, A.; 
Dimier, C.; Picheral, M.; Searson, S.; Kandels-Lewis, S.; Tara Oceans, C.; Acinas, S. G.; Bork, P.; Boss, E.; de Vargas, C.; Gorsky, G.; Ogata, H.; Pesant, S.; Sullivan, M. B.; Sunagawa, S.; Wincker, P.; Karsenti, E.; Bowler, C.; Not, F.; Hingamp, P.; Iudicone, D., Ocean plankton. Environmental characteristics of Agulhas rings affect interocean plankton transport. Science 2015, 348 (6237), 1261447.

25. Li, J.; Jia, H.; Cai, X.; Zhong, H.; Feng, Q.; Sunagawa, S.; Arumugam, M.; Kultima, J. R.; Prifti, E.; Nielsen, T.; Juncker, A. S.; Manichanh, C.; Chen, B.; Zhang, W.; Levenez, F.; Wang, J.; Xu, X.; Xiao, L.; Liang, S.; Zhang, D.; Zhang, Z.; Chen, W.; Zhao, H.; Al-Aama, J. Y.; Edris, S.; Yang, H.; Wang, J.; Hansen, T.; Nielsen, H. B.; Brunak, S.; Kristiansen, K.; Guarner, F.; Pedersen, O.; Dore, J.; Ehrlich, S. D.; Meta, H. I. T. C.; Bork, P.; Wang, J.; Meta, H. I. T. C., An integrated catalog of reference genes in the human gut microbiome. Nat Biotechnol 2014, 32 (8), 834-41.

26. Kanehisa, M.; Goto, S.; Hattori, M.; Aoki-Kinoshita, K. F.; Itoh, M.; Kawashima, S.; Katayama, T.; Araki, M.; Hirakawa, M., From genomics to chemical genomics: new developments in KEGG. Nucleic acids research 2006, 34 (Database issue), D354-7.

27. Kanehisa, M.; Goto, S.; Sato, Y.; Kawashima, M.; Furumichi, M.; Tanabe, M., Data, information, knowledge and principle: back to metabolism in KEGG. Nucleic acids research 2014, 42 (Database issue), D199-205.

28. Powell, S.; Forslund, K.; Szklarczyk, D.; Trachana, K.; Roth, A.; Huerta-Cepas, J.; Gabaldon, T.; Rattei, T.; Creevey, C.; Kuhn, M.; Jensen, L. J.; von Mering, C.; Bork, P., eggNOG v4.0: nested orthology inference across 3686 organisms. Nucleic acids research 2014, 42 (Database issue), D231-9.

29. Cantarel, B. L.; Coutinho, P. M.; Rancurel, C.; Bernard, T.; Lombard, V.; Henrissat, B., The Carbohydrate-Active EnZymes database (CAZy): an expert resource for Glycogenomics. Nucleic acids research 2009, 37 (Database issue), D233-8.

30. Backhed, F.; Roswall, J.; Peng, Y.; Feng, Q.; Jia, H.; Kovatcheva-Datchary, P.; Li, Y.; Xia, Y.; Xie, H.; Zhong, H.; Khan, M. T.; Zhang, J.; Li, J.; Xiao, L.; Al-Aama, J.; Zhang, D.; Lee, Y. S.; Kotowska, D.; Colding, C.; Tremaroli, V.; Yin, Y.; Bergman, S.; Xu, X.; Madsen, L.; Kristiansen, K.; Dahlgren, J.; Wang, J., Dynamics and Stabilization of the Human Gut Microbiome during the First Year of Life. Cell Host Microbe 2015, 17 (5), 690-703. 